\title{
Linear Integer Optimization Model for Two-Stage Guillotine Cutting Stock Problem Using Branch and Bound Method in the Garment Industry
}

\author{
Eman Lesmana a,", Julita Nahar ${ }^{a}$, Annisa D. P. ${ }^{\text {a }}$ \\ ${ }^{a}$ Department of Mathematics, Faculty of Mathematics and Natural Sciences, Universitas Padjadjaran, INDONESIA \\ *Corresponding author: eman.lesmana@unpad.ac.id
}

\begin{abstract}
This paper discusses the Two-Stage Guillotine Cutting Stock Problem (2GCSP) in the garment industry, namely how to determine the two-stage guillotine pattern that is used to cut fabric stocks into several certain size t-shirt materials that are produced based on the demand for each size of the shirt. 2GCSP is modeled in the form of Linear Integer Optimization and finding solutions using the Branch and Bound method. In this paper also presented a Graphical User Interface with Maple software as an interactive tool to find the best fabric stock cutting patterns. The results show that the optimal solution can be determined by solving numerically using the Branch and Bound method and Maple optimization packages. The solution is shown with an illustration of the pattern and the amount of fabric cut based on the pattern.
\end{abstract}

Keywords: Two dimensional stock cutting problems, two-stage guillotine patterns, linear integer programming, branch and bound methods, graphical user interface.

\section{Introduction}

The garment industry is an industry that produces apparel and apparel equipment. One of the raw materials used in the garment industry is fabric. The fabric has a standard size larger than the size of the material used to make items ordered. Therefore, a cutting problem arises which is referred to as the Two Dimensional Cutting Stock Problem. These problems can be classified based on the rules of cutting patterns, one of which is the two-stage guillotine. There are two stages for determining the two-stage guillotine cutting pattern. The first step is to determine the pattern of cutting fabric stock to form several strips. The second stage is to determine the strip pattern to form the required t-shirt material. This 2 GCSP problem was found in the Merch Cons Bandung garment industry in producing T-shirts. The industry must produce several shirt sizes on demand, but with minimum use of fabric stock. To solve this problem, 2GCSP can be modeled in the Linear Integer Optimization formulation with the objective 
function of minimizing the amount of fabric stock cut. To facilitate the industry in finding optimal patterns, applications are needed that can provide these solutions (Genova \& Guliashki, 2011; Wu \& Ge, 2012; Kavun et al., 2014; Widyastiti et al., 2016; Qu et al., 2017; Mikolajkova et al., 2018; Wang et al., 2019).

Based on this description, the problem discussed in this study is how 2GCSP in the garment industry can be stated as a Linear Integer Optimization problem. Then, how is the 2GCSP optimal solution using the Branch and Bound method in a case study in the Merch Cons Bandung garment industry? Finally, how the pattern finder's Graphical User Interface (GUI) provides the introduction (background, objectives, systematics) of your paper.

\section{Literature Review}

\subsection{Branch and Bound Method}

The literature on the branch and bound method can be seen in (Zhou \& Cao, 2013; Jiao et al., 2014; Tian \& Posypkin, 2014; Andrianova et al., 2016; Smirnova \& Voloshinov, 2018).

\subsubsection{Initialization Stage}

For the maximization objective function, specify $Z^{*}=-\infty$. As for the objective minimization function, specify $Z^{*}=\infty$.

\subsubsection{Iteration Stage}

Branching. For example, $x_{j}$ is a noninteger decision variable, $x_{j}=r_{j}, r_{j} \in \mathbf{R}$. Form two subproblems with the addition of constraints $x_{j} \leq\left\lfloor r_{j}\right\rfloor$ for the first subproblem and $x_{j} \geq\left\lfloor r_{j}\right\rfloor+1$ for the second subproblem in the initial integer linear programming model. According to Chinneck (2015), there are several subproblem selection rules to be branched out, as follow.

- Best-first or global-best: choose the subproblem that has the best limit of any subproblem. For the minimization problem, select the subproblem with the smallest limit. For the problem of maximization, select the subproblem with the largest limit.

- Depth-first: select a subproblem that has the best limit of the newly branched subproblem. If there are no subproblems that can be branched on that level, go back one level and branch out on subproblems that have not yet been branched.

- Breadth-first: branch out subproblems in sequence on one level.

Bounding (restrictions). Round down the value of the objective function $(Z)$ for the maximization problem. Instead, round up the value of the objective function for the minimization problem.

Fathoming (disappearance). For each new subproblem, apply the fathoming test as follows.

- Test 1: In the maximization problem, fathom if the limit is $\leq Z^{*}$. Whereas on the minimization problem, fathom if the limit is $\geq Z^{*} . Z^{*}$ is the current incumbent $Z$ value.

- Test 2: Fathom if PL relaxation does not have a viable solution.

- Test 3: Fathom if the optimal solution for PL relaxation is an integer. If this solution is better than the incumbent, then this solution becomes a new incumbent and test 1 is reapplied to subproblems that are not removed with the new $Z^{*}$.

\subsubsection{Optimality Test}

Stop if there are no more subproblems left. The incumbent (prospective optimal solution) that applies is optimal. If not, re-do the next iteration. 


\subsection{Two-Stage Guillotine Cutting Stock Problem (2GCSP)}

Two-Stage Guillotine Cutting Stock Problem (2GCSP) is a problem that arises in the industry when goods in the form of square or rectangular must be cut from raw materials with the two-stage guillotine cutting pattern (Mrad et al., 2013; Chen, et al., 2015). The classification of the deduction of items in 2 GCSP is a regular form. The guillotine pattern is a cutting pattern that starts from one side of the raw material and then continues on the other side. In this problem, there are two stages in determining the cutting pattern. The first stage, determine the cutting pattern where the cutting is done based on the width of the items or the length of the items on the raw material, to produce several strips. The second step is cutting one by one the strip according to the requested item size.

(tahap 2)

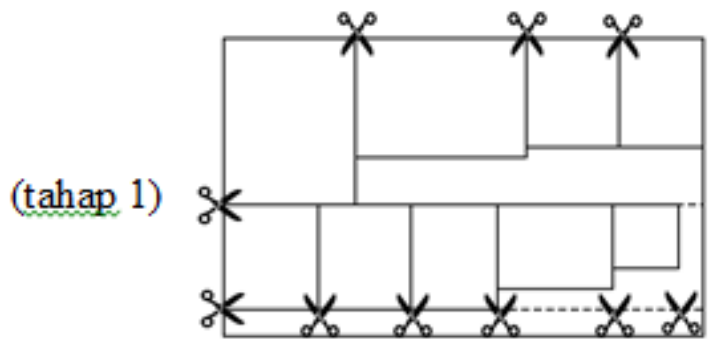

(tahap 2)

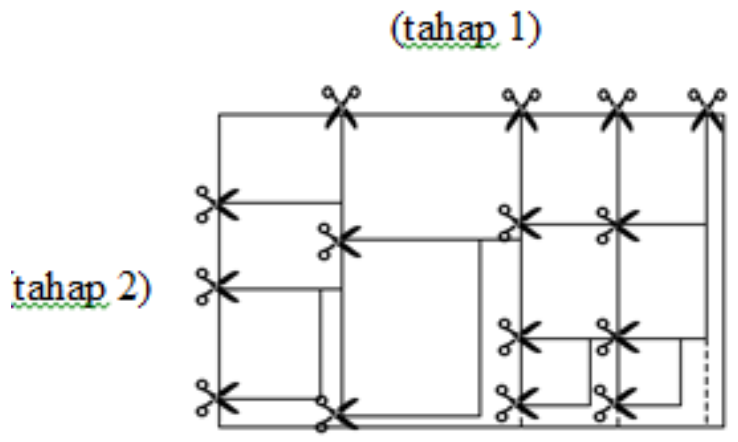

Figure 2: Two-stage guillotine pattern based on length of items

\section{Main Results}

\subsection{GCSP Linear Integer Optimization Model in the Garment Industry}

The following are the assumptions in this model.

- Fabric stock and rectangular T-shirt material.

- The cutting pattern is following the two-stage guillotine pattern with the provisions of the pattern based on the width of the shirt material, the length of the shirt material, and without rotation.

- The optimal cutting pattern is only influenced by the number of requests for each size of shirt, other factors are not taken into account, for example, the price of fabric stock or the price of the shirt.

- The formation of a cut pattern of fabric stock based on the width of the t-shirt material in the first stage starts from the strip with the largest width of the t-shirt material in the fabric stock pattern. While the formation of a cut pattern of fabric stock based on the length of the shirt material in the first stage starts from the strip with the largest length of the shirt material that is in the pattern of the fabric stock.

- Formation of strip cutting patterns based on the width of the t-shirt material in the second stage starts from the t-shirt material with the largest width in the strip pattern and the width of the tshirt material is less than or equal to the width of the strip. Whereas the formation of strip cutting patterns based on the length of the t-shirt material in the second stage starts from the t-shirt material with the largest length in the strip pattern and the length of the t-shirt material is less than or equal to the length of the strip. 
- In the second stage of cut strip pattern, there is at least one T-shirt material that has the same width as the strip width for the cut pattern based on the width of the t-shirt material and at least one T-shirt material that has the same length as the strip length for the cut pattern based on the length of the t-shirt material.

- $\quad$ The cut (the shirt material) may be more than demand.

Table 1: Parameters for the 2GCSP model in the garment industry

\begin{tabular}{|c|c|c|}
\hline No & Notation & Information \\
\hline 1 & $m^{l}$ & the number of different width of the shirt material \\
\hline 2 & $m^{p}$ & the number of different length shirt material \\
\hline 3 & $l_{(i)}$ & width of the smallest $\mathrm{t}$-shirt material $i$-th, $i=1, \ldots, m^{l}$ \\
\hline 4 & $p_{(i)}$ & length of the smallest t-shirt material $i$-th, $i=1, \ldots, m^{p}$ \\
\hline 5 & $\pi^{l}$ & the number of cut patterns of fabric stock based on the width of the shirt material \\
\hline 6 & $\pi^{p}$ & the number of cut patterns of fabric stock based on the length of the shirt material \\
\hline 7 & $\rho_{i}^{l}$ & $\begin{array}{l}\text { the number of strip patterns with } l_{(i)} \text { width, } i=1, \ldots, m^{l} \text {, and length } P \text { cut into rectangles according } \\
\text { to the size of the requested shirt material }\end{array}$ \\
\hline 8 & $\rho_{i}^{p}$ & $\begin{array}{l}\text { the number of strip patterns with } p_{(i)} \text { length, } i=1, \ldots, m^{p} \text {, and width } L \text { cut into rectangles according } \\
\text { to the size of the requested shirt material }\end{array}$ \\
\hline 9 & $a_{i j}^{l}$ & the number of strips with $l_{(i)}$ width in the $j$-cloth cut pattern, $i=1, \ldots, m^{l}, j=1, \ldots, \pi^{l}$ \\
\hline 10 & $a_{i j}^{p}$ & the number of strips with $p_{(i)}$ length in the $j$-cloth cut pattern, $i=1, \ldots, m^{p}, j=1, \ldots, \pi^{p}$ \\
\hline 11 & $b_{s i k}^{l}$ & $\begin{array}{l}\text { the number of type } s \text { t-shirts included in the } k \text {-stripe pattern with } l_{(i)} \text { width, } s \in \mathbf{N}, l_{s} \leq l_{(i)} \text {, } \\
k=1, \ldots \rho_{i}^{l}, i=1, \ldots, m^{l}\end{array}$ \\
\hline 12 & $b_{s i k}^{p}$ & $\begin{array}{l}\text { the number of type } s \text { t-shirts included in the } k \text {-stripe pattern with } p_{(i)} \text { length, } s \in \mathbf{N}, p_{s} \leq p_{(i)} \text {, } \\
k=1, . . \rho_{i}^{p l}, i=1, \ldots, m^{p}\end{array}$ \\
\hline
\end{tabular}

Table 2: Variables for the 2GCSP model in the garment industry

\begin{tabular}{|c|c|c|}
\hline No & Notation & Information \\
\hline 1 & $x_{j}^{l}$ & $\begin{array}{l}\text { the number of fabric stock patterns based on the width of the j-jock material cut in the first stage, } j \\
=1, \ldots, \pi^{l}\end{array}$ \\
\hline 2 & $x_{j}^{p}$ & $\begin{array}{l}\text { the number of fabric stock patterns based on the length of the j-jock cut in the first stage, } j=1, \ldots \text {, } \\
\pi^{p}\end{array}$ \\
\hline 3 & $y_{i k}^{l}$ & $\begin{array}{l}\text { the number of k-strip patterns with the width of } l_{(i)} \text { and length } \mathrm{P} \text { cut in the second step, } k=1, \ldots \rho_{i}^{l} \\
\text { and } i=1, \ldots, m^{l}\end{array}$ \\
\hline 4 & $y_{i k}^{p}$ & $\begin{array}{l}\text { the number of k-strip patterns with } p_{(i)} \text { length and } \mathrm{L} \text { width cut in the second step, } k=1, \ldots \rho_{i}^{p l} \text { and } \\
i=1, \ldots, m^{p}\end{array}$ \\
\hline
\end{tabular}

According to Mrad et al. (2013), the total number of strips with $l_{(i)}$ width in the pattern of fabric stock cut in the first stage is greater or equal to the total number of strip patterns with $l_{(i)}$ width and length $P$ cut in the second stage, so

$$
\sum_{j=1}^{\pi^{l}} a_{i j}^{l} x_{j}^{l} \geq \sum_{k=1}^{\rho_{i}^{l}} y_{i k}^{l}, \quad i=1, \ldots, m^{l}
$$


Then the total number of strips with length $p_{(i)}$ in the pattern of cut fabric stock in the first stage is greater or equal to the total number of strip patterns with length $p_{(i)}$ and width $L$ cut in the second stage, so

$$
\sum_{j=1}^{\pi^{p}} a_{i j}^{p} x_{j}^{p} \geq \sum_{k=1}^{\rho_{i}^{p}} y_{i k}^{p}, \quad i=1, \ldots, m^{p}
$$

Because the strips cut in the second stage must be able to produce t-shirts according to demand and be allowed more than demand, the total amount of production of t-shirt type $s$ is greater or equal to demand so that

$$
\sum_{t \in\{l, p\}} \sum_{i=1}^{m^{t}} \sum_{k=1}^{\rho_{i}^{t}} b_{s i k}^{t} y_{i k}^{t} \geq d_{s}, \quad s=1, \ldots, m .
$$

Then, each decision variable is a non-negative variable and is an integer, it can be written down

$$
\begin{aligned}
& x_{j}^{t} \geq 0, \quad j=1, \ldots, \pi^{t}, t \in\{l, p\}, \\
& y_{i k}^{t} \geq 0, \quad k=1, \ldots, \rho_{i}^{t}, i=1, \ldots, m^{t}, t \in\{l, p\}, \\
& x \text { and } y \text { are integers. }
\end{aligned}
$$

Because the main purpose of this model is to minimize the amount of fabric stock used, the objective function is to minimize the number of cut patterns of fabric stock in the first stage based on the width of the shirt and the length of the shirt. So it can be formulated as follows

$$
\min \sum_{t \in\{l, p\}} \sum_{j=1}^{\pi^{t}} x_{j}^{t}
$$

\subsection{Case Study}

\subsubsection{Data Processing}

Data on the demand for t-shirts production at the Merch Cons Bandung garment industry can be seen in Table 3.

Table 3: Request t-shirts size $\mathrm{S}, \mathrm{M}, \mathrm{L}$ and $\mathrm{XL}$

\begin{tabular}{cc}
\hline T-shirts Size & Demand (pieces) \\
\hline S & 24 \\
M & 24 \\
L & 36 \\
XL & 24 \\
\hline
\end{tabular}

The raw materials used to produce these shirts are combed 20s cotton fabric with a length of $200 \mathrm{~cm}$ and a width of $100 \mathrm{~cm}$. The standard size of the $\mathrm{t}$-shirt material used at Merch Cons is the USA international standard size with details of the size of each $\mathrm{t}$-shirt that is in Table 4. 
Table 4: US International Size Standards for t-shirt materials

\begin{tabular}{ccccc}
\hline $\begin{array}{c}\text { T-shirts } \\
\text { Size }\end{array}$ & $\begin{array}{c}\text { Body Material } \\
\text { Length }(\mathbf{c m})\end{array}$ & $\begin{array}{c}\text { Body Material } \\
\text { Width }(\mathbf{c m})\end{array}$ & $\begin{array}{c}\text { Length of Arm Section } \\
\text { Material }(\mathbf{c m})\end{array}$ & $\begin{array}{c}\text { Sleeve Material } \\
\text { Width }(\mathbf{c m})\end{array}$ \\
\hline S & 70 & 46 & 43 & 22 \\
M & 72 & 51 & 48 & 24.5 \\
L & 75 & 56 & 52.5 & 25 \\
XL & 77 & 61 & 57.5 & 28 \\
\hline
\end{tabular}

To make one shirt, two pieces of body parts are needed, namely the front and back of the shirt so that the material requirements for making each size of shirt can be seen in Table 5 .

Table 5: Request for each material

\begin{tabular}{lc}
\hline \multicolumn{1}{c}{ Material } & Request (Sheets) \\
\hline Sleeve material size $\mathrm{S}\left(S_{l}\right)$ & 48 \\
Sleeve material size $\mathrm{M}\left(M_{l}\right)$ & 48 \\
Sleeve material size L $\left(L_{l}\right)$ & 72 \\
Sleeve material size $\mathrm{XL}\left(X L_{l}\right)$ & 48 \\
Body material size $\mathrm{S}\left(S_{b}\right)$ & 48 \\
Body material size M $\left(M_{b}\right)$ & 48 \\
Body material size L $\left(L_{b}\right)$ & 72 \\
Body material size XL $\left(X L_{b}\right)$ & 48 \\
\hline
\end{tabular}

\subsubsection{Problem Solving}

First, determine all cutting patterns of the first and second stages that are possible. The following is an illustration of the first and second stage cutting patterns based on the width of the shirt material and the length of the shirt material.

\section{$P$}

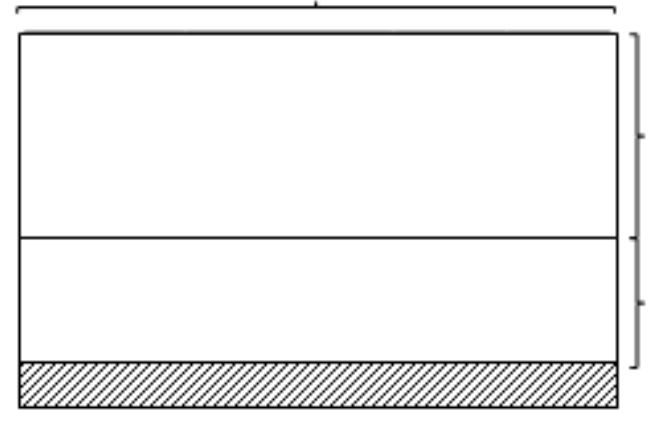

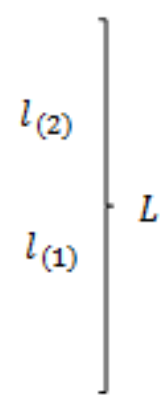

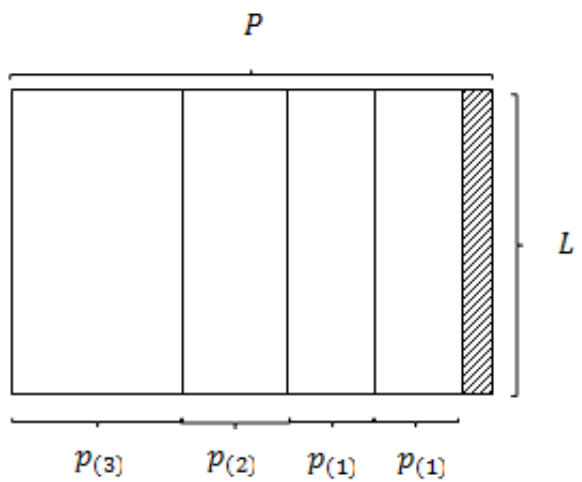

Figure 3: Illustration of first stage pattern based on shirt width and shirt length 

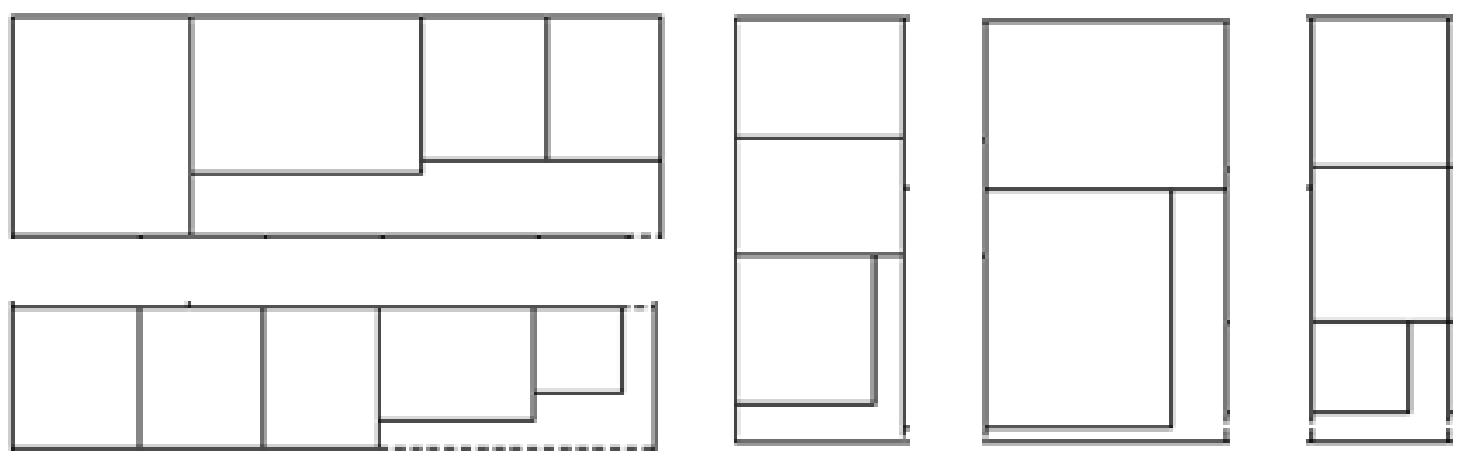

Figure 4: Illustration of second stage pattern based on shirt width and shirt length

By determining the pattern, the values of the parameters can be determined so that the 2GCSP problem in the Merch Cons Bandung garment industry can be stated in the Linear Integer Optimization model.

Then, here are the steps to find a Linear Integer Optimization model solution with Maple software based on the Branch and Bound method.

- Type restart to delete data that has been previously input as follows.

$>$ restart:

- Use the optimization and linalg packages in the worksheet by typing as follows.

$>$ with (linalg) :

$>$ with(Optimization) :

- The form of the first stage cutting pattern matrix is based on the width of the $\mathrm{t}$-shirt material $\left(a_{i j}^{l}\right)$ where each element is a constraint coefficient 1-8 with the following syntax. matrix $\left.\left(\| a_{11}^{l}, \ldots, a_{1 j}^{l}\right] \ldots, \mid a_{81}^{l}, \ldots, a_{8 j}^{l} \|\right)$

- The form of the first stage cutting pattern matrix is based on the length of the shirt material $\left(\left[a_{i j}^{p}\right)\right.$ where each element is a 9-16 constraint coefficient with the following syntax.

matrix $\left(\left[a_{11}^{p}, \ldots, a_{1 j}^{p}\right], \ldots,\left\{a_{81}^{p}, \ldots, a_{8 j}^{p}\right\rceil\right)$

- The constraint coefficient matrix form 17-24 is based on the second stage cutting pattern with the following syntax.

matrix $\left.\| b_{1,1,1}^{l}, \ldots, b_{1,8,18}^{l} b_{1,1,1}^{p}, \ldots, b_{1,8,4}^{p}\right] \ldots,\left[b_{81,1,1}^{l}, \ldots, b_{8,8,18}^{l} b_{8,1,1}^{p}, \ldots, b_{8,8,4}^{p}\right] \mid$

- The $\mathbf{Y}$ matrix form as a constraint variable 17-24 as follows.

$Y:=\operatorname{matrix}([[y l[1][1]], \operatorname{seq}([y l[2][i]], i=1 . .4), \operatorname{seq}([y l[3][i]], i=1$

$. .7), \operatorname{seq}([y l[4][i]], i=1 . .10), \operatorname{seq}([y l[5][i]], i=1 . .14)$,

$\operatorname{seq}([y l[6][i]], i=1 . .17), \operatorname{seq}([y l[7][i]], i=1 . .18)$,

$\operatorname{seq}([y l[8][i]], i=1 . .18),[y p[1][1]], \operatorname{seq}([y p[2][i]], i=1 . .4)$,

$\operatorname{seq}([y p[3][i]], i=1 . .10), \operatorname{seq}([y p[4][i]], i=1 . .10)$,

$\operatorname{seq}([y p[5][i]], i=1 . .10), \operatorname{seq}([y p[6][i]], i=1 . .6)$,

$\operatorname{seq}([y p[7][i]], i=1 . .4), \operatorname{seq}([y p[8][i]], i=1 . .4)])$

- Create the left side constraint function by using for as follows. 
for $i$ from 1 to 8 do kendala $[i]:=\sum_{j=1} A L[i, j] \cdot x l[j]$ od:

$>$ for $i$ from 1 to 8 do kendala $2[i]:=\sum_{j=1}^{89} A P[i, j] \cdot x p[j]$ od:

$>$ for $i$ from 1 to 8 do kendala $3[i]:=\sum_{j=1}^{138} B[i, j] \cdot Y[j, 1]$ od:

- Create the right hand segment function as follows.

$$
\begin{aligned}
&> T D L[1]:=y\left[[1][1]: T D L[2]:=\sum_{k=1}^{4} y l[2][k]: T D L[3]\right. \\
&:=\sum_{k=1}^{7} y\left[[3][k]: T D L[4]:=\sum_{k=1}^{10} y l[4][k]: T D L[5]\right. \\
&:=\sum_{k=1}^{14} y\left[[5][k]: T D L[6]:=\sum_{k=1}^{17} y l[6][k]: T D L[7]\right. \\
&:=\sum_{k=1}^{18} y\left[[7][k]: T D L[8]:=\sum_{k=1}^{18} y l[8][k]:\right. \\
&>T D P[1]:=y p[1][1]: T D P[2]:=\sum_{k=1}^{4} y p[2][k]: T D P[3] \\
&:=\sum_{k=1}^{10} y p[3][k]: T D P[4]:=\sum_{k=1}^{10} y p[4][k]: T D P[5] \\
&:=\sum_{k=1}^{10} y p[5][k]: T D P[6]:=\sum_{k=1}^{6} y p[6][k]: T D P[7] \\
&:=\sum_{k=1}^{4} y p[7][k]: T D P[8]:=\sum_{k=1}^{4} y p[8][k]: \\
&>d[1]:=48: d[2]:=48: d[3]:=72: d[4]:=48: d[5]:=48: d[6] \\
&:=48: d[7]:=72: d[8]:=48:
\end{aligned}
$$

- For the initialization phase, set $Z 1=\infty$ and determine the solution of relaxation LP with the following syntax.

$Z 1:=$ infinity

$$
\begin{aligned}
& \text { solusi }:=\text { convert }\left(\text { LPSolve } \left(\sum_{j=1}^{49} x l[j]+\sum_{j=1}^{89} x p[j],[\text { seq }(\text { kendala }[m]\right.\right. \\
& \quad \geq T D L[m], m=1 \ldots 8), \text { seq }(\text { kendala } 2[m] \geq T D P[m], m=1 . .8), \\
& \text { seq }(\text { kendala }[n] \geq d[n], n=1 . .8)], \text { assume }=\text { nonnegative }), \\
& \text { rational })
\end{aligned}
$$

- For the iteration step, do the Branch and Bound algorithm, complete the relaxation LP in each subproblem by adding a new obstacle to the syntax solution in point 9.

The following solutions can be provided for the Merch Cons Bandung garment industry using the Branch and Bound method with variable selection rules that are natural order that has noninteger values and subproblem selection rules namely Depth First Selection (DFS).

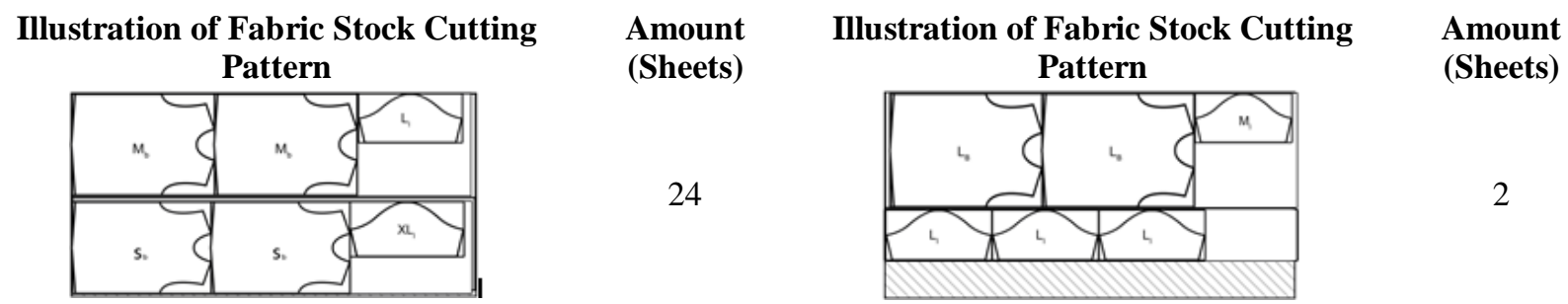



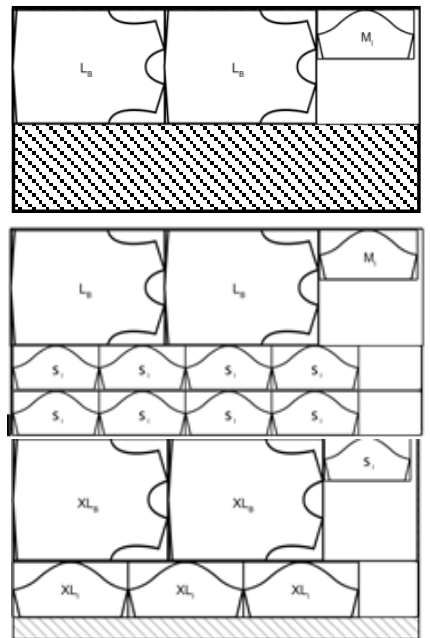

30

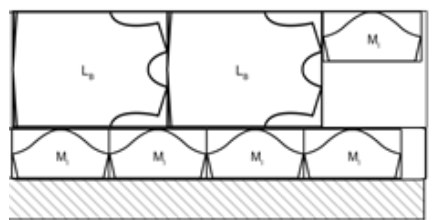

3
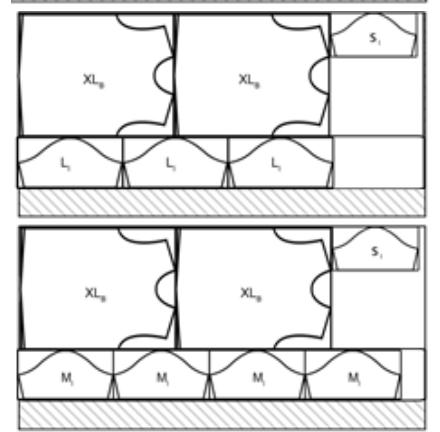

1

14

2

Thus, the number of T-shirt materials produced with the cut pattern is given in Table 6 .

Table 6: The Number of t-shirt materials produced

\begin{tabular}{lc}
\hline \multicolumn{1}{c}{ Material } & Stock (Sheets) \\
\hline Sleeve material size $\mathrm{S}\left(S_{l}\right)$ & 48 \\
Sleeve material size $\mathrm{M}\left(M_{l}\right)$ & 48 \\
Sleeve material size L $\left(L_{l}\right)$ & 72 \\
Sleeve material size XL $\left(X L_{l}\right)$ & 48 \\
Body material size S $\left(S_{b}\right)$ & 48 \\
Body material size M $\left(M_{b}\right)$ & 48 \\
Body material size L $\left(L_{b}\right)$ & 72 \\
Body material size XL $\left(X L_{b}\right)$ & 48 \\
\hline
\end{tabular}

\subsection{GUI Finders of Optimum Cutting Patterns on Fabric Stocks}

In the GUI created there are 2 input choices. The first is the t-shirt size data input and the second is the t-shirt material demand data input as shown in Figure 5. 

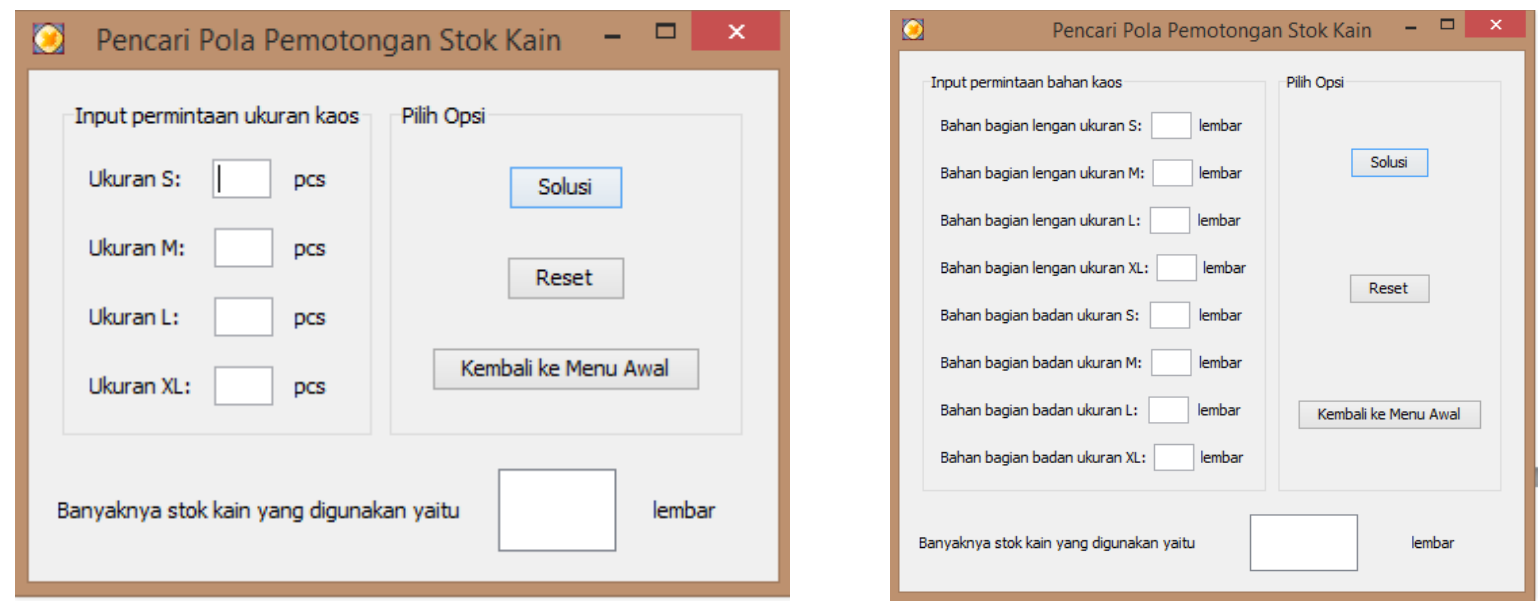

Figure 5: Input window

For example, enter the t-shirt size data in the Merch Cons Garment industry in Bandung. Then after clicking the solution, the results of the amount of stock of cloth used will appear in the textbox. Details of the pattern of the first and second stages can be seen in the Microsoft Excel file as follows. 


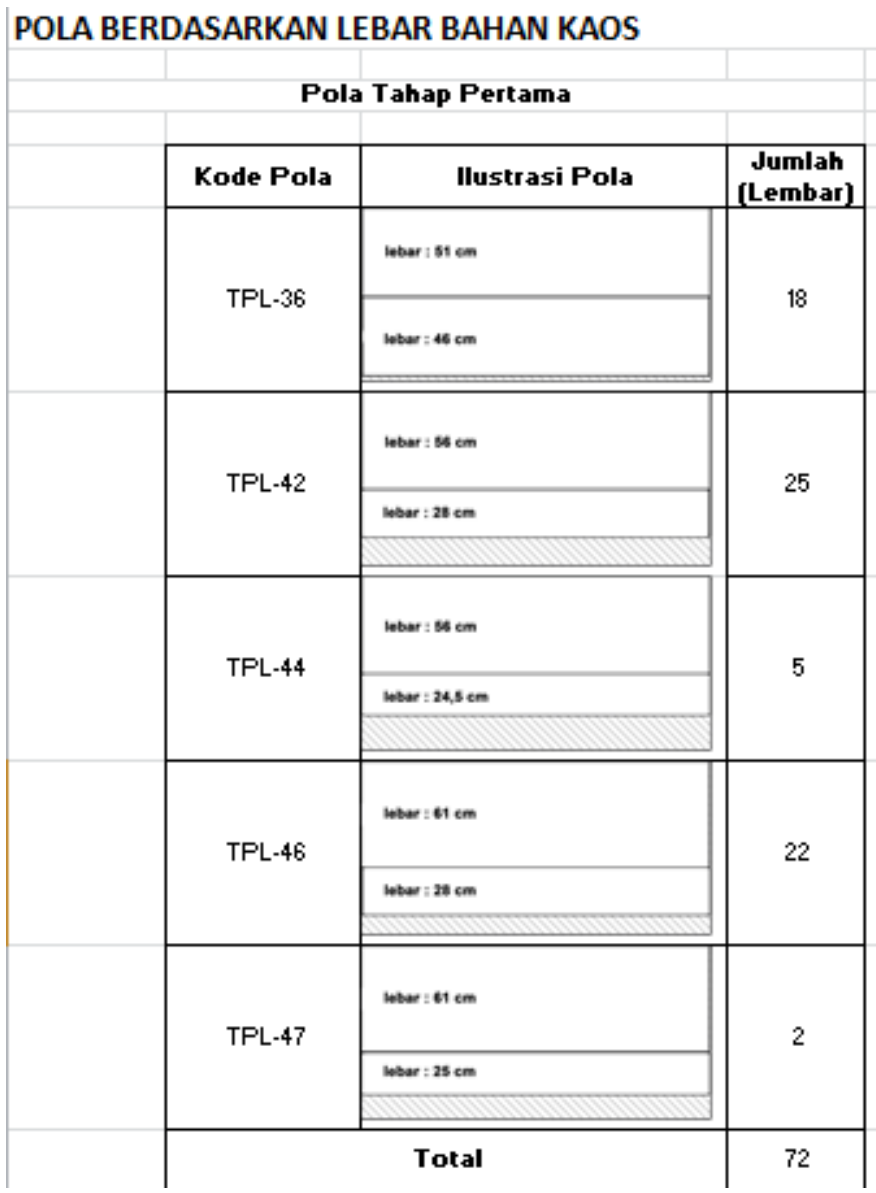

POLA BERDASARKAN PANJANG BAHAN KAOS

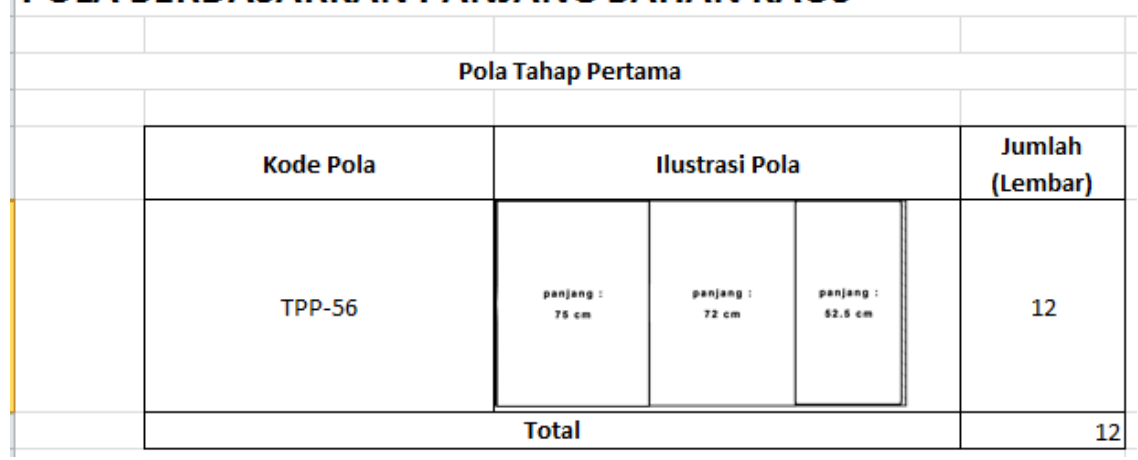






Figure 6: Detailed patterns of first and second stage in Microsoft Excel

\section{Conclussion}

Two-Stage Guillotine Cutting Stock Problem (2GCSP) in the garment industry can be stated in the Linear Integer Optimization model with the objective function of minimizing the amount of fabric stock cut and constraints namely cutting pattern rules based on t-shirt widths, cutting pattern rules based on tshirt lengths, and pattern rules the second stage is based on the request of the shirt. The optimal solution of 2GCSP in case studies in the Merch Cons Bandung garment industry can be determined using the Branch and Bound method. The method provides an integer value solution for each decision variable. The solution is an optimal pattern of cutting fabric stock in the first and second stages where the pattern of cutting fabric stock can meet the demand of each size of the shirt. Graphical User Interface (GUI) to find optimal cutting patterns can be made using Maple software. The GUI is used as an interactive tool 
for users to input t-shirt production data in the garment industry. The result is an optimal stage one and stage two cutting pattern in a Microsoft Excel file. These results are the solution of the 2GCSP Linear Integer Optimization model that has been made based on the Optimization package on Maple software.

\section{References}

Andrianova, A. A., Korepanova, A. A. \& Halilova, I. F. (2016). One algorithm for branch and bound method for solving concave optimization problem. IOP Conf. Series: Materials Science and Engineering, 158, pp. 012005.

Chen, Q. L., Li, L. P., Cui, Y. D., Chen, Y. \& Lu, X. Y. (2015). A Heuristic for the 3-staged 2D Cutting Stock Problem with Usable Leftover. International Conference of Electrical, Automation and Mechanical Engineering, pp. 776-779.

Chinneck, J. W. (2015). Practical Optimization: a Gentle Introduction. (This version dated June 23, 2015 and available online at www.sce.carleton.ca/faculty/chinneck/po.html)

Genova, K. \& Guliashki, V. (2011). Linear Integer Programming Methods and Approaches - A Survey. Cybernetics and Information Technologies, 11(1), pp. 3-25.

Jiao, H., Wang, F. \& Chen, Y. (2014). An Effective Branch and Bound Algorithm for Minimax Linear Fractional Programming. Journal of Applied Mathematics, 2014, 8 pages.

Kavun, S., Daradkeh, Y. I., Aldhaifallah, M. (2014). Method of the Integer Linear Programming. Mitteilungen Klosterneuburg, 64, pp. 1-13.

Mikolajkova, M., Saxen, H. \& Pettersson, F. (2018). Mixed Integer Linear Programming Optimization of Gas Supply to a Local Market. Ind. Eng. Chem. Res., 57, pp. 5951-5965.

Mrad, M., Meftahi, I. \& Haouari, M. (2013). A branch-and-price algorithm for the two-stage guillotine cutting stock problem. Journal of the Operational Research Society, 64, pp. 629-637.

Qu, X., Yi, W., Wang, T., Wang, S., Xiao, L. \& Liu, Z. (2017). Mixed-Integer Linear Programming Models for Teaching Assistant Assignment and Extensions. Scientific Programming, 2017, 7 pages.

Smirnova, S. \& Voloshinov, V. (2018). On Domain Decomposition Strategies to Parallelize Branch-and-Bound Method for Global Optimization in Everest Distriburted Environment. Procedia Computer Science, 136, pp. 128-135.

Tian, B. \& Posypkin, M. (2014). Efficient Implementation of Branch-and-Bound Method on Desktop Grids. Computer Science, 15(3), pp. 239-252.

Wang, L., Ni, M., Gao, J., Shen, Q., Jia, Y. \& Yao, C. (2019). The Loading Optimization: A Novel Integer Linear Programming Model. Enterprise Information Systems. doi: 10.1080/17517575.2019.1631964

Widyastiti, M., Aman, A. \& Bakhtiar, T. (2016). Nurses Scheduling by Considering the Qualification using Integer Linear Programming. Telkomnika, 14(3), pp. 933-940.

Wu, J. \& Ge, X. (2012). Optimization Research of Generation Investment Based on Linear Programming Model. Physics Procedia, 24, pp. 1400-1405.

Zhao, X. \& Cao, B. (2013). A Simplicial Branch and Bound Duality-Bounds Algorithm to Linear Multiplicative Programming. Journal of Applied Mathematics, 2013, 10 pages. 